\title{
Mecanismos de corrosión en materiales compuestos de matriz de aluminio con refuerzo de SiC
}

\author{
A. UREÑA, E. OTERO, M.V. UTRILLA, P. RODRIGO \\ Dpto. de Tecnología Química, Ambiental y de los Materiales. \\ E. S. Ciencias Experimentales y Tecnología. \\ Universidad Rey Juan Carlos. 28933 Móstoles (Madrid). España
}

\begin{abstract}
Los materiales compuestos estudiados están constituidos por una aleación AA2009 reforzada con un 15\% en volumen de SiC en forma de whiskers y de partículas. Mediante técnicas de polarización cíclica se compara el comportamiento de ambos materiales, en estado de recepción y sometidos a tratamientos térmicos, frente a la corrosión por picadura en disolución acuosa desaireada de $\mathrm{NaCl}$ al 3,5\% en peso; determinándose el potencial de picadura $\left(\mathrm{E}_{\mathrm{pic}}\right)$, el potencial de corrosión $\left(\mathrm{E}_{\text {corr }}\right)$ y la densidad de corriente $\left(\mathrm{i}_{\text {corr }}\right)$. Ambos materiales presentaron un potencial de picadura muy similar. También se han llevado a cabo medidas electroquímicas para evaluar el $\mathrm{E}_{\text {corr }}$ y la $\mathrm{i}_{\text {corr }}$ en función de tiempo de exposición. Los valores de $\mathrm{E}_{\text {corr }}$ obtenidos son más nobles en el caso de la aleación sin reforzar que para el material compuesto, lo que indica un mejor comportamiento en este medio. La caracterización de los materiales y de los mecanismos que rigen la corrosión se ha realizado mediante microscopía de barrido (SEM).
\end{abstract}

Palabras clave: Material compuesto de matriz de aluminio, SiC, corrosión.

Corrosion mechanisms of aluminium- silicon carbide composites

Tested composites were constituted by an AA2009 (Al-Cu-Mg) aluminium alloy reinforced with $15 \%$ of SiC, studding two different of morphologies: particles and whiskers. Cyclic polarisation measurements were done in a deaerated aqueous solution of $\mathrm{NaCl}(3,5 \mathrm{weight}$ $\%$ ) on both kind of composites, for comparative purposes. The following properties were determined from these corrosion tests: pitting potential $\left(\mathrm{E}_{\text {pit }}\right)$, corrosion potential $\left(\mathrm{E}_{\text {corr }}\right)$ and corrosion density $\left(\mathrm{i}_{\text {corr }}\right) \cdot \mathrm{E}_{\text {pit }}$ is similar for both materials. Electrochemical tests were performed to evaluate $\mathrm{E}_{\text {corr }}$ and $\mathrm{i}_{\text {corr }}$ versus time. The corrosion potential is more noble for the aluminium alloy without reinforcement. Finally, materials were observed by SEM to characterize the corrosion mechanisms.

Key words: Aluminum matrix composites, SiC, corrosion.

\section{INTRODUCCIÓN}

Los materiales compuestos de matriz metálica normalmente se diseñan para que ofrezcan unas buenas propiedades mecánicas, aunque tengan baja resistencia a la degradación ambiental. En general, los materiales compuestos de matriz de aluminio reforzados con $\mathrm{SiC}$ son más susceptibles al ataque por corrosión que sus componentes por separado (1). Aunque el aluminio es un metal extremadamente reactivo, normalmente presenta una buena resistencia a la corrosión en disolución acuosa debido a la formación de su capa pasiva de óxido. En tales medios el $\mathrm{SiC}$ puede actuar como electrodo inerte, y puede existir corrosión galvánica (2). Además, los intermetálicos desarrollados en las aleaciones de aluminio en los tratamientos térmicos pueden influir de forma negativa en el comportamiento frente a la corrosión del material $(3,4)$.

\section{PROCEDIMIENTO EXPERIMENTAL}

\subsection{Materiales de ensayo}

Para el presente estudio se emplearon dos materiales compuestos constituidos por la misma matriz, una aleación de aluminio AA2009 (Al-Cu-Mg), reforzados con un $15 \%$ de $\mathrm{SiC}$ con dos morfologías distintas, partículas $(\mathrm{SiCp})$ y whiskers $(\mathrm{SiCw})$; fabricados por Advanced Composites Materials Cooporation (ACMC). Como material de referencia se ha utilizado una aleación comercial de aluminio, AA2024 sin reforzar, cuya composición en los elementos de aleación mayoritarios es similar a la de la matriz de los materiales compuestos utilizados (tabla I).

TABLA I: COMPOSICIÓN NOMINAL DE LAS ALEACIONES DE ALUMINIO.

\begin{tabular}{|c|c|c|c|c|c|c|}
\hline Aleación & $\mathbf{C u}$ & $\mathbf{M g}$ & $\mathbf{Z n}$ & $\mathbf{S i}$ & $\mathbf{F e}$ & Al \\
\hline AA2009 & $3,2-4,4$ & $1,0-1,6$ & 0,1 & 0,25 & 0,07 & Resto \\
\hline AA2024 & 4,47 & 1,53 & 0,12 & 0,26 & 0,36 & Resto \\
\hline
\end{tabular}

Las muestras para los ensayos electroquímicos se embutieron en resina epoxi de baja contracción y se desbastaron hasta granulometría 600. Las que iban a ser observadas mediante SEM se pulieron con pasta de diamante hasta $0,1 \mu \mathrm{m}$.

Sobre algunas muestras de material compuesto se realizó un tratamiento de envejecimiento natural (T4), consistente en la solubilización del material a $500{ }^{\circ} \mathrm{C}$ durante 2 horas, temple en hielo y maduración a temperatura ambiente.

\subsection{Ensayos Electroquímicos}

Para la realización de estos ensayos se utilizó un potenciostatogalvanostato Autolab PGSTAT 30. La celda electroquímica utilizada consistía en un electrodo de referencia $\mathrm{Ag} / \mathrm{AgCl}$, grafito como contraelectrodo y el electrodo de trabajo. El medio corrosivo en todos los casos fue una disolución al 3,5\% en masa de $\mathrm{NaCl}$. 
En primer lugar, para estudiar la influencia que tiene la presencia del refuerzo en la resistencia a la corrosión de una aleación de aluminio, se realizaron medidas electroquímicas de corriente continua mediante los métodos de resistencia de polarización y de intersección. Los ensayos tuvieron una duración de 14 días, tomándose medidas cada 24 horas.

Para determinar la influencia de la morfología del refuerzo se hicieron medidas sobre los materiales compuestos, en estado de recepción y con un tratamiento T4. Los ensayos de picadura se realizaron en la disolución desaireada durante 30 minutos. Se ha utilizado el método del doble ciclo de polarización, DCP (5). El $\mathrm{E}_{\text {corr }}$ se determinó, tras una estabilización de una hora, antes de desairear la disolución. Como $\mathrm{E}_{\mathrm{pic}}$ se tomó el punto en el que se producía una variación brusca de la pendiente de la curva para pequeñas variaciones de potencial.

\section{RESULTADOS Y DISCUSIÓN}

Para minimizar el efecto del procesado en los resultados se trabajó con una sección paralela a la dirección de extrusión, en la que los granos son alargados y el refuerzo presenta direccionalidad, apareciendo en la matriz bandas de mayor o menor porcentaje de refuerzo, también se observaron agrupaciones locales de refuerzo (clusters).

\subsection{Medidas electroquímicas de velocidades de corrosión.}

La figura 1 (a) muestra la variación de la densidad de corriente $\left(i_{\text {corr }}\right)$ en función del tiempo de ensayo. Se puede observar que $i_{\text {corr }}$ presenta valores muy bajos en los tres materiales, lo que indica un buen comportamiento a la corrosión en este medio. En cuanto al $\mathrm{E}_{\text {corr' }}$ la aleación sin reforzar presenta valores mayores que el de los materiales compuestos; entre éstos no existe mucha diferencia, tal como se aprecia en la figura 1 (b). La adición de refuerzo cerámico, por tanto, hace que los materiales compuestos sean menos nobles que la aleación $\sin$ reforzar.

Si se observa la superficie de las muestras ensayadas se aprecia que la aleación sin reforzar presenta una corrosión más generalizada, mayor número de picaduras de pequeño tamaño (figura 2); sin embargo, los materiales compuestos presentan menor número de picaduras pero de mayor tamaño (figura 3), lo que indica que se produce un ataque más localizado que en la aleación sin reforzar.

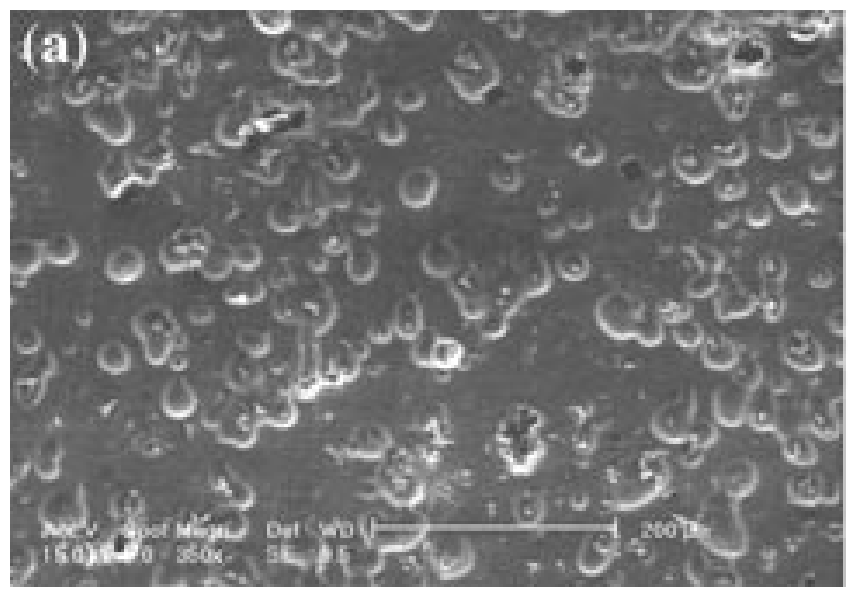

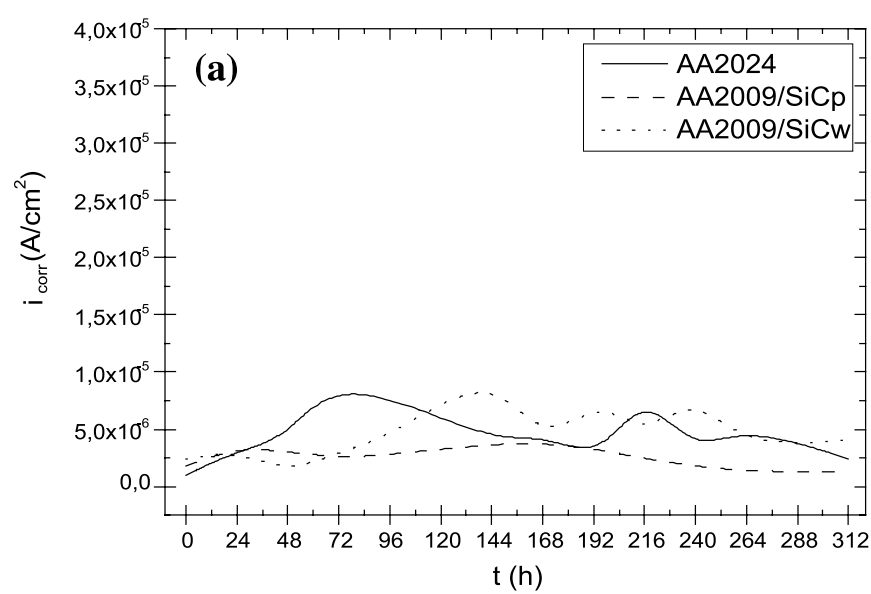

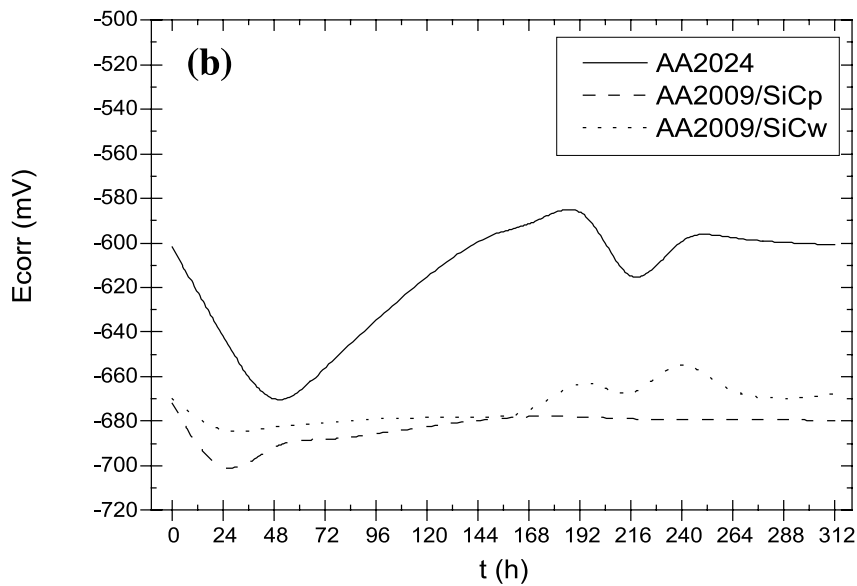

Figura 1: (a) Variación de la $i_{\text {corr }}$ con el tiempo de ensayo (b) Variación del $\mathrm{E}_{\text {corr }}$ con el tiempo de ensayo

\subsection{Medidas electroquímicas de polarización cíclica}

En las curvas obtenidas al realizar los ensayos con una doble polarización se observa que los valores de $\mathrm{E}_{\text {corr }} \mathrm{y}$ de $\mathrm{E}_{\text {pic' }}$ recogidos en la tabla II, están muy próximos entre sí. En todos los casos, tanto en la aleación con el refuerzo en forma $\mathrm{SiCp}$ como en forma de $\mathrm{SiCw}$, se observaba la aparición de una zona de pasivación en la curva (aumento del potencial sin variación importante de la densidad de corriente) debida a la presencia de la capa superficial de óxido. A potenciales en

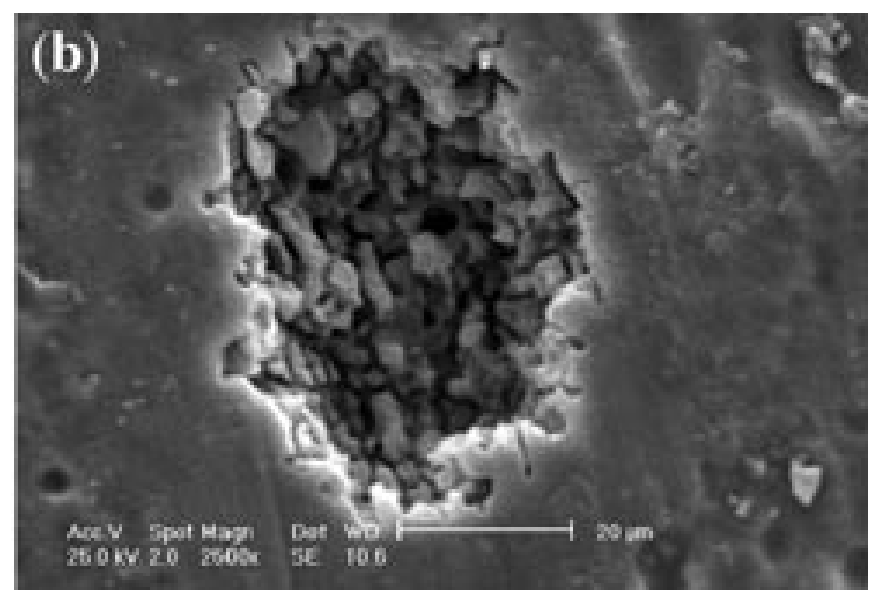

Figura 2: (a) AA2024 después de 14 días de ensayo (b) Detalle de una de las picaduras. 

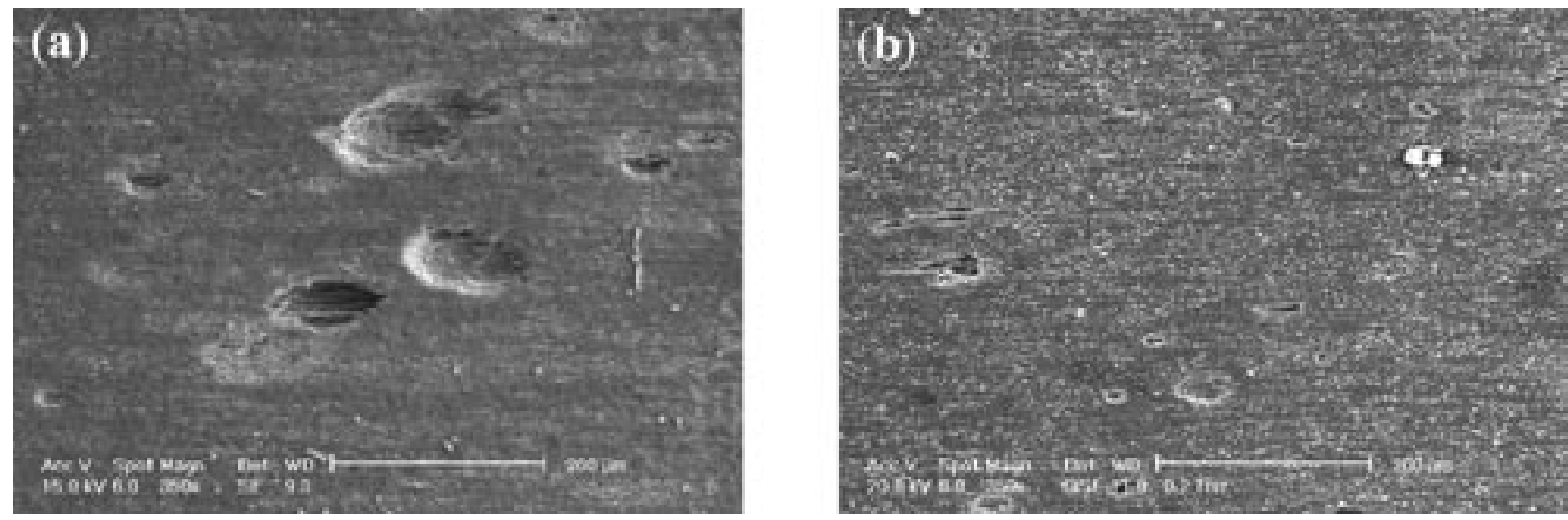

Figura 3: Materiales compuestos después de 14 días de ensayo (a) AA2009/SiCp (b) AA2009/SiCw
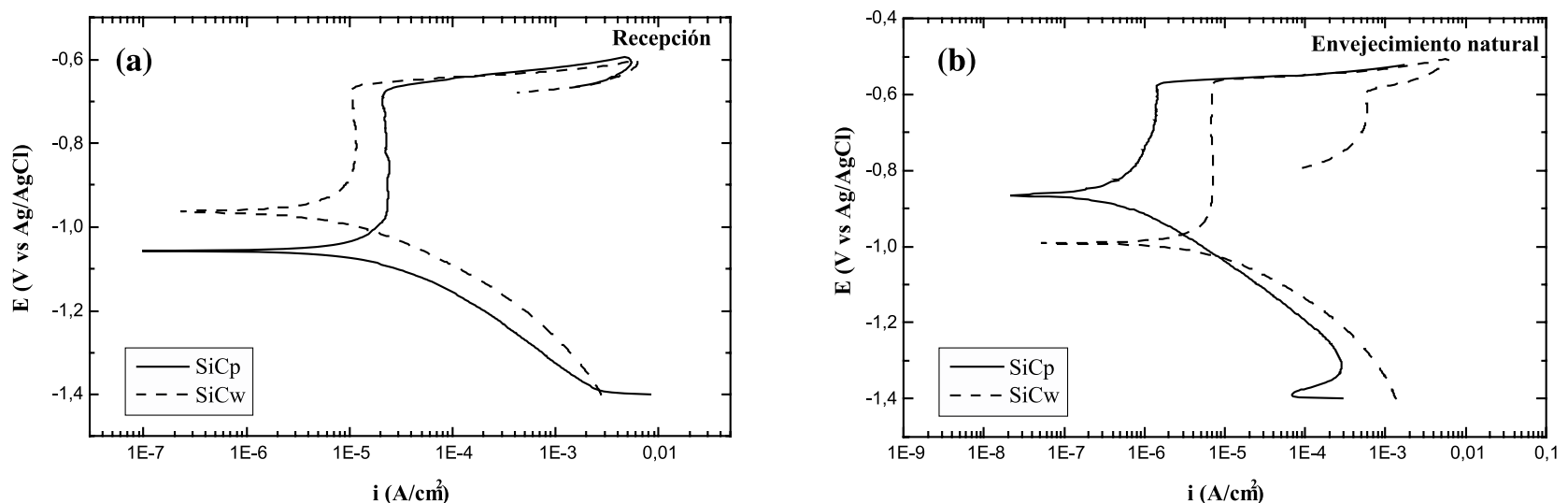

Figura 4: Curvas de polarización del $2^{\circ}$ ciclo del material (a) en estado de recepción y (b) con tratamiento T4

torno a $-680 \mathrm{mV}$, aparecía un brusco aumento de la densidad de corriente originado por la rotura de la película protectora al nuclearse las picaduras (figura 4). Por lo tanto, la forma del refuerzo no produce una variación apreciable en el material con respecto al valor del $\mathrm{E}_{\mathrm{pic}} \cdot$

TABLA II: ECORR Y EPIC OBTENIDOS EN LOS ENSAYOS DE AA2009/SIC

\begin{tabular}{|l|l|c|c|}
\hline \multicolumn{2}{|c|}{ Material } & $\mathbf{E}_{\text {corr }}(\mathbf{m V})$ & $\mathbf{E}_{\text {pic }}(\mathbf{m V})$ \\
\hline \multirow{2}{*}{ Recepción } & $\mathrm{SiCp}$ & -685 & -681 \\
\cline { 2 - 4 } & $\mathrm{SiCw}$ & -675 & -676 \\
\hline \multirow{2}{*}{ Envejec. Natural } & $\mathrm{SiCp}$ & -599 & -573 \\
\cline { 2 - 4 } & $\mathrm{SiCw}$ & -583 & -560 \\
\hline
\end{tabular}

Cuando los materiales eran sometidos a un tratamiento T4 y ensayados de la misma forma que el de recepción, se observó que las curvas de polarización se desplazan hacia valores menores de densidad de corriente, presentando, además, valores de $\mathrm{E}_{\mathrm{pic}}$ más nobles, lo que indica una mejora de la resistencia a la corrosión de los materiales. Esto es debido a la reducción de zonas activas, como son los intermetálicos de equilibrio que, preferentemente, aparecen rodeando al refuerzo. Las curvas correspondientes a la aleación reforzada con $\mathrm{SiCp}$ aparecen desplazadas a valores de densidades de corriente más bajas que con los $\mathrm{SiCw}$, por lo que, aunque la picadura se inicia a un potencial similar en ambos materiales, la velocidad de corrosión en el material reforzado con whiskers es mayor que en el reforzado con SiCp. Esta diferencia de comportamiento puede deberse a la morfología del refuerzo, ya que es el único factor de diferencia en los materiales tratados.
Mediante SEM se comprobó que también existían diferencias en el estado de la matriz. Las SiCp tienen un tamaño que oscila entre 2-4 $\mu \mathrm{m}$; apreciándose la presencia de gran cantidad de precipitados, dispersos por la matriz y sobre todo en la intercara. $\mathrm{Los} \mathrm{SiCw}$ son monocristales con forma de prisma hexagonal, con base de aproximadamente $1,5 \mu \mathrm{m}$ de diámetro y entre 5 y $20 \mu \mathrm{m}$ de longitud. La presencia de precipitados en este material es mucho menor. Estos precipitados son en su mayoría de fase $\theta\left(\mathrm{Al}_{2} \mathrm{Cu}\right)$, pero también aparece fase $\mathrm{S}\left(\mathrm{Al}_{2} \mathrm{CuMg}\right)$, nucleando ambos preferentemente en la intercara matriz/ $\mathrm{SiC}$.

El hecho de que el material reforzado de partículas en estado de recepción presente más cantidad de intermetálicos que el de whiskers puede explicar que este último tenga mejor comportamiento frente a la corrosión por picadura. Teniendo en cuenta los potenciales de corrosión de aleaciones de aluminio $\mathrm{Al}-\mathrm{Cu}$, en $\mathrm{NaCl} 0,1 \mathrm{M}(4), \mathrm{el}_{2} \mathrm{Cl}_{2} \mathrm{Cu}$ es más noble que el aluminio puro y que una aleación $\mathrm{Al}-2 \% \mathrm{Cu}$ y menos que una aleación $\mathrm{Al}-4 \% \mathrm{Cu}$. Los precipitados de $\left(\mathrm{Al}_{2} \mathrm{CuMg}\right)$ actuarían siempre como ánodo, disolviéndose.

Las zonas de matriz cercanas a los precipitados de $\mathrm{Al}_{2} \mathrm{Cu}$ tendrán, por tanto, un contenido en cobre menor del $4 \%$, ya que este elemento por difusión ha pasado a formar la fase $\theta^{\prime}$, con lo que dichas zonas actuarán como ánodo frente a los precipitados, disolviéndose. Cuanto más cantidad de fase $\theta$ tenga el material, existirán más zonas de matriz con bajo contenido en Cu por lo que, a su vez, habrá más zonas susceptibles de inicio de picadura. Sin embargo, los precipitados de $\mathrm{Al}_{2} \mathrm{CuMg}$ actúan como ánodo frente a la matriz y frente $\mathrm{al}_{2} \mathrm{Cu}$. Por ese motivo, en cualquier caso, la presencia de mayor cantidad de intermetálicos está asociada con una mayor cantidad de lugares de nucleación de picaduras. 

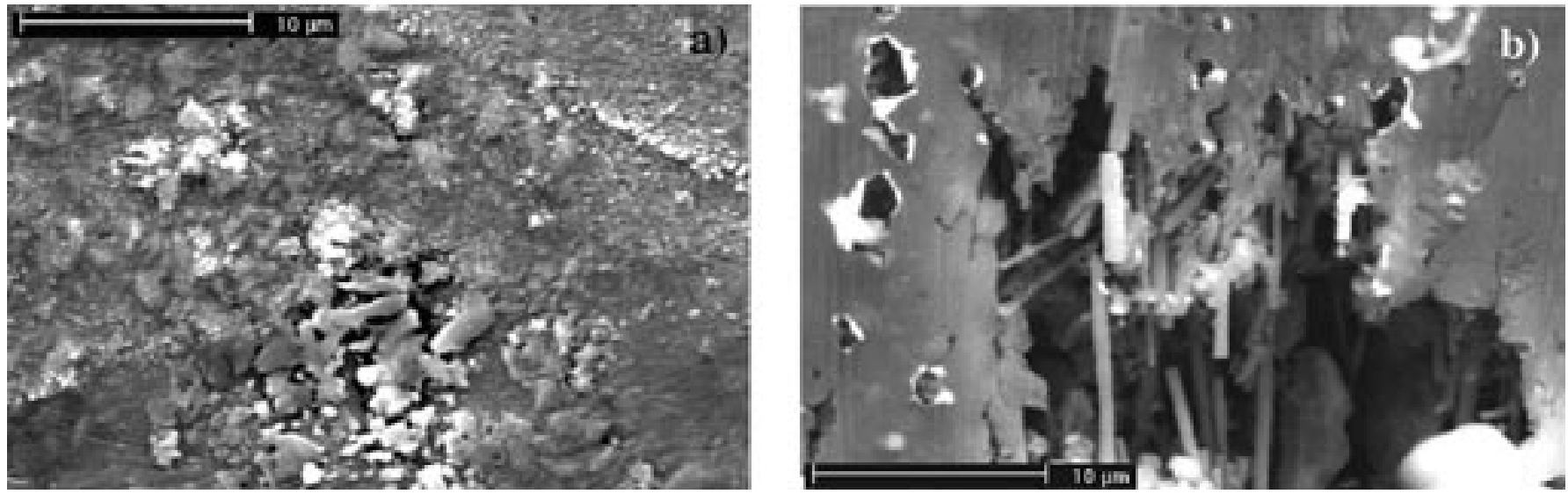

Figura 5 (a) Iniciación de una picadura en AA2009/SiCp; (b) Picadura de mayor tamaño sobre AA2009/SiCw

La iniciación de la picadura tendrá lugar en zonas de acumulación de refuerzo (clusters), que es donde también se concentra la mayoría de los intermetálicos. En unos casos, nuclearían en la intercara $\mathrm{Al}_{2} \mathrm{Cu}$ / matriz, quedando la fase $\theta$ adherida al refuerzo, y en otros se disolvería la intercara $\mathrm{SiC} / \mathrm{Al}_{2} \mathrm{CuMg}$, quedando la superficie del $\mathrm{SiC}$ limpia de precipitados. Una vez nucleada la picadura puede avanzar por las intercaras matriz/refuerzo limpias de intermetálicos, debido a que las discontinuidades en los materiales son lugares preferentes para el avance de la corrosión.

La figura 5 muestra dos imágenes de SEM realizadas en muestras ensayadas. En 5 (a) se muestra la nucleación de una picadura en el material de recepción de partículas, apreciándose como el ataque avanza alrededor del refuerzo y de la fase $\theta$ (en blanco). En 5 (b) aparece una picadura en el material de whiskers que demuestra que el ataque nuclea y avanza preferentemente en zonas de clusters de refuerzo, distinguiéndose como sobre la superficie de los $\mathrm{SiCw}$ han quedado los intermetálicos de $\mathrm{Al}_{2} \mathrm{Cu}$ sin disolver.

\section{CONCLUSIONES}

1. Los tres materiales presentan velocidades de corrosión similares y muy bajas, lo que indica un buen comportamiento en el medio de estudio. Los potenciales de corrosión en los materiales reforzados muestran valores más negativos, lo que demuestra una peor resistencia a la corrosión que la aleación sin reforzar.

2. Los dos materiales presentaron un potencial de picadura muy similar. Las diferentes densidades de corriente observadas están relacionadas con dos factores: el estado de tratamiento que presenta la matriz de aluminio y la morfología del refuerzo.
3. El tratamiento térmico de envejecimiento natural mejora la resistencia a la corrosión, ya que el potencial de picadura se desplaza hacia valores más nobles, debido a la reducción de zonas activas en el material.

4. La formación de intermetálicos sobre la superficie del $\mathrm{SiC}$ provoca que la nucleación de las picaduras se produzca, principalmente, en la intercara matriz/intermetálico. El avance de la picadura se produce a través de las demás intercaras, por lo que las grandes picaduras están asociadas con zonas de acumulación de refuerzo (clusters).

\section{AGRADECIMIENTOS}

Los autores agradecen al MCYT (proyecto MAT 2000-1646-C02-01) y a la Universidad Rey Juan Carlos (PGRAL-2001/13) la financiación proporcionada.

\section{BIBLIOGRAFÍA}

1. T.M. Yue, S.M. Mei, K.C. Chan, H.C. Man. "The effect of excimer laser surface treatment on corrosion resistance of aluminium 2009/SiCw composite". Materials Letters 39 (1999) 274-279.

2. T.M. Yue, Y.X. Wu, H.C.Man. "On the role of $\mathrm{CuAl}_{2}$ precipitates in pitting corrosion of aluminum 2009/SiCw metal matrix composites". Journal of Materials Science Letters 19, 2000, 1003-1006.

3. Z. Szklarska-Smialowska. "Pitting corrosion of aluminum". Corrosion Science 41 (1999) 1743-1767.

4. J.F.McIntre, R.K.Conrad, S.L.Golledge. “Technical Note: The effect of heat treatment on the pitting behavior of SiCw/AA2124". Corrosion, Vol. 46, No. 11, pp.902-905, 1990.

5. Grigoris E Kiourtsidis, Stefanos M. Skolianos, Eleni G. Pavlidou. “A study on pitting of AA2024/SiCp composites using the double cycle polarization technique". Corrosion Science 41 (1999) 1185-1203. 